\title{
A note on Helson's conjecture on moments of random multiplicative functions
}

\author{
Adam J. Harper*, Ashkan Nikeghbali, and Maksym Radziwiłł
}

To Prof. Helmut Maier on the occasion of his sixtieth birthday

\section{Introduction}

In this note we are interested in cancellations in sums of multiplicative functions. It is well known that

$$
M(x):=\sum_{n \leq x} \mu(n)=O\left(x^{1 / 2+\varepsilon}\right)
$$

is equivalent to the Riemann Hypothesis. On the other hand it is also a classical result that $M(x)>x^{1 / 2-\varepsilon}$ for a sequence of arbitrarily large $x$. It is in fact conjectured that

$$
\varlimsup_{x \rightarrow \infty} \frac{M(x)}{\sqrt{x}(\log \log \log x)^{\frac{5}{4}}}= \pm B
$$

for some constant $B>0$ (see [21]).

Adam J. Harper

Jesus College, Cambridge CB5 8BL, England, e-mail: A.J.Harper@dpmms .cam.ac.uk

Ashkan Nikeghbali

University of Zürich, Institute of Mathematics, Winterthurerstrasse 190, CH-8057 Zürich, e-mail: ashkan.nikeghbali@math.uzh.ch

Maksym Radziwiłł

Department of Mathematics, Rutgers University, Hill Center for the Mathematical Sciences, 110 Frelinghuysen Rd., Piscataway, NJ 08854-8019, e-mail:maksym.radziwill@gmail.com

* The first author is supported by a research fellowship at Jesus College, Cambridge. 
Wintner [24] initiated the study of what happens for a generic multiplicative function which is as likely to be 1 or -1 on the primes. Consider $f(p)$, a sequence of independent random variables taking values \pm 1 with probability $1 / 2$ each (i.e. Rademacher random variables), and define a multiplicative function supported on squarefree integers $n$ by

$$
f(n):=\prod_{p \mid n} f(p) .
$$

We shall refer to such a function as a Rademacher random multiplicative function. By the three series theorem, the Euler product $F(s):=\prod_{p}\left(1+f(p) p^{-s}\right)$ converges almost surely for $\Re s>\frac{1}{2}$. From this Wintner deduced that

$$
\sum_{n \leq x} f(n) \ll x^{1 / 2+\varepsilon} \text { almost surely (a.s.) }
$$

Since then the problem of the behavior of $\sum_{n \leq x} f(n)$ has attracted considerable attention [7, 11, 12, 13, 17, 18]. A closely related model is to let $f(p)$ be uniformly distributed on the complex unit circle (i.e. Steinhaus random variables), and then define $f(n):=\prod_{p^{\alpha} \| n} f(p)^{\alpha}$ for all $n$. We shall refer to such a function as a Steinhaus random multiplicative function.

Very recently mean values of random multiplicative functions arose in connection with harmonic analysis. In his last paper Helson [16] considered the question of generalizing Nehari's theorem to the infinite polydisk. He noticed that the generalization could be disproved if one could show that

$$
\lim _{T \rightarrow \infty} \frac{1}{T} \int_{0}^{T}\left|\sum_{n \leq N} n^{-i t}\right| d t=o(\sqrt{N}) .
$$

Using Bohr's identification, we have

$$
\left(\mathbb{E}\left|\sum_{n \leq N} f(n)\right|^{2 q}\right)^{1 / 2 q}=\left(\lim _{T \rightarrow \infty} \frac{1}{T} \int_{0}^{T}\left|\sum_{n \leq N} n^{-i t}\right|^{2 q} d t\right)^{1 / 2 q}
$$

for all $2 q>0$, and with $f(n)$ a Steinhaus random multiplicative function. Therefore (1) is equivalent to

$$
\mathbb{E}\left|\sum_{n \leq N} f(n)\right|=o(\sqrt{N})
$$

with $f(n)$ a Steinhaus random multiplicative function. Helson justified his belief in (11) by observing that $N(i t):=\sum_{n \leq N} n^{-i t}$ is the multiplicative analogue of the classical Dirichlet kernel $D(\theta):=\sum_{|n| \leq N} e^{2 \pi i n \theta}$. Since $\|D\|_{1}=o\left(\|D\|_{2}\right)$ Helson conjectured that the same phenomenon should happen for the "multiplicative analogue" $N(i t)$. Another reason one might believe the large cancellation in (1) to be possible is that on the $\frac{1}{2}$-line one has 


$$
\lim _{T \rightarrow \infty} \frac{1}{T} \int_{0}^{T}\left|\sum_{n \leq N} \frac{1}{n^{1 / 2+i t}}\right| d t \ll \log ^{1 / 4+o(1)} N,
$$

as follows from the work of Bondarenko, Heap and Seip [2]. This bound is stronger than one would expect assuming only squareroot cancellation, which would suggest a size more like $\log ^{1 / 2} N$.

Recently Ortegà-Cerda and Seip [22] proved that Nehari's theorem doesn't extend to the infinite polydisk. However the problem of establishing (1) remained. There are now reasons to believe that (1) is false. In a recent paper Bondarenko and Seip [3] showed that the first absolute moment is at least $\sqrt{N}(\log N)^{-\delta+o(1)}$ for some small $\delta<1$. Our primary goal in this note is to improve further on the lower bounds for (2). Our results also work for Rademacher random multiplicative functions.

Theorem 1. Let $f(n)$ be a Rademacher or Steinhaus random multiplicative function. Then,

$$
\mathbb{E}\left|\sum_{n \leq N} f(n)\right| \gg \sqrt{N}(\log \log N)^{-3+o(1)}
$$

as $N \rightarrow \infty$.

The main input in the proof of Theorem 1 is the work [12] of the first named author on lower bounds for sums of random multiplicative functions. Using Hölder's inequality, we can extend the result of Theorem 1 to $L^{q}$ norms.

Theorem 2. Let $f(n)$ be a Rademacher or Steinhaus random multiplicative function and let $0 \leq q \leq 1$. Then,

$$
\mathbb{E}\left|\sum_{n \leq N} f(n)\right|^{2 q} \gg N^{q}(\log \log N)^{-6+o(1)} .
$$

Theorem 1 and Theorem 2 suggest it is rather unlikely that Helson's conjecture is true. See Conjecture1 below.

In addition to the above results, we establish an asymptotic estimate for the $2 k$-th moment when $k$ is a positive integer.

Theorem 3. Let $k \in \mathbb{N}$. Suppose that $f(n)$ is a Steinhaus random multiplicative function. Then, as $N \rightarrow \infty$,

$$
\mathbb{E}\left|\sum_{n \leq N} f(n)\right|^{2 k} \sim\left(\begin{array}{c}
2 k-2 \\
k-1
\end{array}\right) k^{-(k-1)} \cdot c_{k} \gamma_{k} \cdot N^{k} \cdot(\log N)^{(k-1)^{2}} .
$$

where $\gamma_{k}$ is the volume of Birkhoff polytope $\mathscr{B}_{k}$, defined as the $(k-1)^{2}$ dimensional volume of the set of $\left(u_{i, j}\right) \in \mathbb{R}_{+}^{k^{2}}$ such that, 


$$
\begin{aligned}
\text { for each } i \leq k: \sum_{1 \leq j \leq k} u_{i, j} & =1 \\
\text { and for each } j \leq k: \sum_{1 \leq i \leq k} u_{i, j} & =1,
\end{aligned}
$$

and

$$
c_{k}=\prod_{p}\left(1-\frac{1}{p}\right)^{k^{2}} \cdot\left(1+\sum_{\alpha \geq 1} \frac{\left(\begin{array}{c}
\alpha+k-1 \\
k-1
\end{array}\right)^{2}}{p^{\alpha}}\right) .
$$

Note that $\mathscr{B}_{k}$ is a $(k-1)^{2}$ dimensional object embedded in a $k^{2}$ dimensional space. The $(k-1)^{2}$ dimensional volume of $\mathscr{B}_{k}$ is equal (see e.g. section 2 of Chan and Robbins [6]) to $k^{k-1}$ times the full-dimensional volume of the set of $\left(u_{i, j}\right)_{i, j \leq k-1} \in$ $\mathbb{R}^{(k-1)^{2}}$ such that, for all $i, j \leq k-1$,

$$
\sum_{j \leq k-1} u_{i, j} \leq 1 \text { and } \sum_{i \leq k-1} u_{i, j} \leq 1 \text { and } \sum_{i, j \leq k-1} u_{i, j} \geq k-2 .
$$

The latter is how the volume of $\mathscr{B}_{k}$ will actually arise in our calculations.

It is worth pointing out that finding a closed formula for the volume of the Birkhoff polytope $\mathscr{B}_{k}$ is a notorious open question and would be of interest in enumerative combinatorics, statistics and computational geometry (see [23]). There are evaluations of $\operatorname{Vol}\left(\mathscr{B}_{k}\right)$ for small values of $k$, (see [1] and [6]),

$$
\operatorname{Vol}\left(\mathscr{B}_{3}\right)=\frac{3 \cdot 3^{2}}{2^{2} !}, \operatorname{Vol}\left(\mathscr{B}_{4}\right)=\frac{352 \cdot 4^{3}}{3^{2} !}, \operatorname{Vol}\left(\mathscr{B}_{5}\right)=\frac{4718075 \cdot 5^{4}}{4^{2} !}, \ldots
$$

and an asymptotic formula is known to hold [5]

$$
\operatorname{Vol}\left(\mathscr{B}_{k}\right) \sim \sqrt{2 \pi} e^{1 / 3} \cdot \frac{k^{-(k-1)^{2}} e^{k^{2}}}{(2 \pi)^{k}}, k \rightarrow \infty .
$$

In addition the asymptotic behavior of the Euler product $c_{k}$ is known (see [9, Proposition]),

$$
\log c_{k}=-k^{2} \log \left(2 e^{\gamma} \log k\right)+o\left(k^{2}\right)
$$

where $\gamma$ is the Euler-Mascheroni constant.

We note that Conrey and Gamburd [8] compute the even integer moments

$$
\lim _{T \rightarrow \infty} \frac{1}{T} \int_{0}^{T}\left|\sum_{n \leq N} n^{-1 / 2-i t}\right|^{2 k} d t
$$

on the $\frac{1}{2}$-line, and unsurprisingly the answer is extremely similar to Theorem 3 (in particular an Euler product and a volume related to the Birkhoff polytope again appear). Conrey and Gamburd discuss the connection between their result and the moments of certain truncated characteristic polynomials of random matrices. In general, it seems reasonable to say that the arithmetic factor $c_{k}$ reflects local counting modulo different primes in the moment computation, whereas the geometric factor 
$\gamma_{k}$ reflects global counting of tuples $n_{1}, \ldots, n_{k}$ and $m_{1}, \ldots, m_{k}$ subject to the truncation $n_{i}, m_{i} \leq N$.

We deduce Theorem 3 from a general result of La Bretèche [4] on mean values of multiplicative functions in several variables. Theorem 3 has also been obtained independently by Granville and Soundararajan (unpublished), and also very recently (and independently) by Heap and Lindqvist [15]. Additionally, Theorem 3 sheds light on the conjectural behavior of moments of the theta functions,

$$
\theta(x, \chi)=\sum_{n \geq 1} \chi(n) e^{-\pi n^{2} x / p}
$$

with $p \geq 3$ a prime, and $\chi$ an even Dirichlet character modulo $p$. The rapidly decaying factor $e^{-\pi n^{2} x / p}$ essentially restricts the sum to those $n$ less than about $\sqrt{p}$ (if $x=1$, say), and the average behavior of $\chi(n)$ with $n \ll p^{1 / 2}$ is similar to that of a Steinhaus random multiplicative function. Therefore Theorem 3 leads to the conjecture that

$$
\frac{1}{p} \sum_{\chi \bmod p}|\theta(1, \chi)|^{2 k} \sim C_{k} p^{k / 2}(\log p)^{(k-1)^{2}} \quad \text { as } p \rightarrow \infty .
$$

In unpublished recent work the same conjecture was stated by Marc Munsch on the basis of his lower bound for moments of $\theta(1, \chi)$. Louboutin and Munsch [19] prove the conjecture for $k=1$ and $k=2$.

Combining Theorem 2 and Theorem 3 suggests the following "counter-conjecture" to Helson's claim (1).

Conjecture 1 If $f(n)$ is a Steinhaus random multiplicative function, then we have as $N \rightarrow \infty$,

$$
\mathbb{E}\left|\sum_{n \leq N} f(n)\right|^{2 q} \sim \begin{cases}C(q) N^{q}, & \text { for } 0 \leq q \leq 1 \\ C(q) N^{q}(\log N)^{(q-1)^{2}}, & \text { for } 1 \leq q .\end{cases}
$$

Conjecture 1 1 suggests a possible line of attack on the problem of showing that for a positive proportion of even characters $\chi$ modulo $p$, we have $\theta(1, \chi) \neq 0$. This would be based on comparing the first and second absolute moments, i.e

$$
\sum_{\chi \bmod p}|\theta(1, \chi)| \text { and } \sum_{\chi \text { even }}|\theta(1, \chi)|^{2} .
$$

We emphasise that we do not have a lot of evidence towards Conjecture 1 when $q \notin \mathbb{N}$, and perhaps especially when $0<q<1$, and it is conceivable the behaviour could be more complicated. However this is the simplest possible conjecture respecting the information that we now have. In addition for $q>1$ it perhaps seems unlikely that the distribution of the tails of $\sum_{n<N} f(n)$ (in a large deviation regime) fluctuates so significantly that it would affect the exponent $(q-1)^{2}$ of the logarithm 
when $q$ goes from an integer to a fractional exponent. We also note that if we could obtain the order of magnitude for the $2 q$-th moment suggested by the Conjecture 1 for $q=\frac{1}{2}$, then since we know it trivially for $q=1$ a simple argument using Hölder's inequality (as in the proof of Theorem 2 , below) would establish the order of magnitude suggested by the Conjecture for all $0 \leq q \leq 1$.

Finally, following a question from the referee, we noticed that we can extend Theorem 3 to the Rademacher case. We omit the simple cases of $k=1,2$ in the theorem below, since both are different from the case $k \geq 3$.

Theorem 4. Let $f(n)$ be a Rademacher random multiplicative function. Then, for $k \geq 3$ an integer, as $N \rightarrow \infty$,

$$
\mathbb{E}\left(\sum_{n \leq N} f(n)\right)^{k} \sim C_{k} \cdot N^{k / 2}(\log N)^{k(k-3) / 2}
$$

with $C_{k}>0$ constant.

Similarly as in Theorem 3 the constant $C_{k}$ splits into an arithmetic and geometric factor. The interested reader should have no trouble working out the details. Theorem 4 has also been obtained independently by Heap and Lindqvist [15].

At first glance it may seem strange that all the moments here (including the odd ones) are non-trivially large, but that is because in the Rademacher case there is no distinction between a term and its complex conjugate (and similarly if one calculated an expression like $\mathbb{E}\left|\sum_{n \leq N} f(n)\right|^{2 k}\left(\sum_{n \leq N} f(n)\right)$ in the Steinhaus case, this would be non-trivially large provided $k \geq 1$ ). Note also that the moments are rather larger in the Rademacher case than the Steinhaus case, again because everything is real valued and so the terms exhibit less cancellation.

Acknowledgments We are grateful to the referee for a careful reading of the paper and for asking several questions which led to Theorem 4 and stronger results in Theorem 3.

\section{Lower bounds for the first moment}

In this section we shall first prove the following result.

Proposition 1 Let $f(n)$ be a Rademacher random multiplicative function. There exist arbitrarily large values of $x$ for which

$$
\mathbb{E}\left|\sum_{n \leq x} f(n)\right| \geq \frac{\sqrt{x}}{(\log \log x)^{3+o(1)}} .
$$

The same is true if $f(n)$ is a Steinhaus random multiplicative function.

The above proposition is actually a fairly straightforward deduction from the work of Harper [12]. However, it is a bit unsatisfactory because it only gives a lower 
bound along some special sequence of $x$ values. With more work we can correct this defect, as in the following theorem announced in the Introduction:

Theorem 1 Let $f(n)$ be a Rademacher random multiplicative function. Then for all large $x$ we have

$$
\mathbb{E}\left|\sum_{n \leq x} f(n)\right| \geq \frac{\sqrt{x}}{(\log \log x)^{3+o(1)}} .
$$

The same is true if $f(n)$ is a Steinhaus random multiplicative function.

The proof of Proposition 1 has two ingredients. The first is the observation, essentially due to Halász [11], that one can almost surely lower bound an average of $\left|\sum_{n \leq x} f(n)\right|$ in terms of the behaviour of $f(n)$ on primes only: more specifically, in the Rademacher case we almost surely have that, for any $y \geq 2$,

$$
\int_{1}^{\infty} \frac{\left|\sum_{n \leq z} f(n)\right|}{z^{3 / 2+1 / \log y}} d z \gg \sup _{t \geq 1} \exp \left(\sum_{p} \frac{f(p) \cos (t \log p)}{p^{1 / 2+1 / \log y}}-\log t-\log \log (t+2) / 2\right) .
$$

Here the implicit constant in the $\gg$ notation is absolute. The reader should note that the presence of the supremum over $t$ will be very significant here, since at any fixed $t$ the expected size of the right hand side would be too small to produce a useful result (about $\log ^{1 / 4} y$, rather than about $\log y$ which is what we need).

The second ingredient is a strong lower bound for the expected size of the right hand side, which we deduce from the work of Harper [12]. We quote the relevant statements from Harper's work as a lemma now, for ease of reference later.

Lemma 1 (See $\S 6.3$ of [12].) If $(f(p))_{p \text { prime }}$ are independent Rademacher random variables, then with probability $1-o(1)$ as $x \rightarrow \infty$ we have

$$
\begin{aligned}
\sup _{1 \leq t \leq 2(\log \log x)^{2}} \sum_{p} \frac{f(p) \cos (t \log p)}{p^{1 / 2+1 / \log x} \geq \log \log x-} \log \log \log x & \\
& -O\left((\log \log \log x)^{3 / 4}\right) .
\end{aligned}
$$

If $(f(p))_{\text {p prime }}$ are independent Steinhaus random variables, then with probability $1-o(1)$ as $x \rightarrow \infty$ we have

$$
\begin{aligned}
& \sup _{1 \leq t \leq 2(\log \log x)^{2}} \sum_{p}\left(\frac{\Re\left(f(p) p^{-i t}\right)}{p^{1 / 2+1 / \log x}}+\frac{1}{2} \frac{\Re\left(f(p)^{2} p^{-2 i t}\right)}{p^{1+2 / \log x}}\right) \geq \log \log x-\log \log \log x \\
& -O\left((\log \log \log x)^{3 / 4}\right) .
\end{aligned}
$$

The first statement here is proved in the last paragraph in $\S 6.3$ of [12] (noting that the quantity $y$ there is $\log ^{8} x$ ). The second statement can be proved by straightforward adaptation of that argument, the point being that the expectation and covariance structure of these random sums in the Steinhaus case are the same, up to negligible error terms, as in the Rademacher case, so the same arguments can be applied. (See the preprint [14] for an explicit treatment of some very similar Steinhaus random 
sums.) The argument in [12] is quite involved, but the basic aim is to show that, for the purpose of taking the supremum, the sums $\sup _{1 \leq t \leq 2(\log \log x)^{2}} \sum_{p} \frac{f(p) \cos (t \log p)}{p^{1 / 2+1 / \log x}}$ behave somewhat independently at values of $t$ that are separated by $\gg 1 / \log x$, so one has something like the supremum over $\log x$ independent samples.

To prove Theorem 1 we introduce a third ingredient, namely we show that $\mathbb{E}\left|\sum_{n \leq x} f(n)\right|$ may itself be lower bounded in terms of an integral average of $\mathbb{E}\left|\sum_{n \leq z} f(n)\right|$, as follows:

Proposition 2 Let $f(n)$ be a Rademacher random multiplicative function. For any large $x$ we have

$$
\mathbb{E}\left|\sum_{n \leq x} f(n)\right| \gg \frac{\sqrt{x}}{\log x} \int_{1}^{\sqrt{x}}\left(\frac{\mathbb{E}\left|\sum_{n \leq z} f(n)\right|}{\sqrt{z}}\right) \frac{d z}{z} .
$$

The same is true if $f(n)$ is a Steinhaus random multiplicative function.

This uses the multiplicativity of $f(n)$ in an essential way (as does the proof of Proposition 1 , of course).

Theorem 1 then follows quickly by combining Proposition 2 with the proof of Proposition 1.

As the reader will see, the proof of Proposition 2 is based on a "physical space" decomposition of the sum $\sum_{n \leq x} f(n)$, which is somewhat related to the martingale arguments of Harper [13]. This is unlike the other arguments above, which work by establishing a connection between the integral average of $\sum_{n \leq x} f(n)$ and its Dirichlet series $\sum_{n} f(n) / n^{s}$ (on the "Fourier space" side).

\subsection{Proof of Proposition 1}

The proof of Proposition 1 is slightly cleaner in the Rademacher case, because then $f(p)^{2} \equiv 1$ for all primes $p$. So we shall give the proof in that case first, and afterwards explain the small changes that arise in the Steinhaus case.

We know from work of Wintner [24] that almost surely $\sum_{n \leq x} f(n)=O_{\varepsilon}\left(x^{1 / 2+\varepsilon}\right)$. Consequently, by partial summation the Dirichlet series $F(s):=\sum_{n} f(n) / n^{s}$ is almost surely convergent in the half plane $\Re(s)>1 / 2$, and then by term by term integration it satisfies

$$
F(s)=s \int_{1}^{\infty} \frac{\sum_{n \leq z} f(n)}{z^{s+1}} d z, \quad \Re(s)>1 / 2 .
$$

In particular, $F(s)$ is almost surely a holomorphic function on the half plane $\Re(s)>$ $1 / 2$.

On the other hand, since $f(n)$ is multiplicative we have for any $\Re(s)>1$ that, in the Rademacher case, 


$$
\begin{aligned}
F(s)=\prod_{p}\left(1+\frac{f(p)}{p^{s}}\right)=\exp \left(\sum_{p} \log \left(1+\frac{f(p)}{p^{s}}\right)\right) \\
=\exp \left(\sum_{p} \frac{f(p)}{p^{s}}-\frac{1}{2} \sum_{p} \frac{f(p)^{2}}{p^{2 s}}+\sum_{k \geq 3} \frac{(-1)^{k+1}}{k} \sum_{p} \frac{f(p)^{k}}{p^{k s}}\right) .
\end{aligned}
$$

Therefore in the Rademacher case we have

$$
s \int_{1}^{\infty} \frac{\sum_{n \leq z} f(n)}{z^{s+1}} d z=\exp \left(\sum_{p} \frac{f(p)}{p^{s}}-\frac{1}{2} \sum_{p} \frac{f(p)^{2}}{p^{2 s}}+\sum_{k \geq 3} \frac{(-1)^{k+1}}{k} \sum_{p} \frac{f(p)^{k}}{p^{k s}}\right)
$$

at least when $\Re(s)>1$, since both sides are equal to $F(s)$. But all the sums involving $p^{2 s}$ and $p^{k s}$ are clearly absolutely convergent whenever $\Re(s)>1 / 2$, and therefore define holomorphic functions there. In addition, for any fixed $s$ with $\Re(s)>1 / 2$ the series $\sum_{p} \frac{f(p)}{p^{s}}$ is a sum of independent random variables, and Kolmogorov's Three Series Theorem implies it converges almost surely. Since a Dirichlet series is a holomorphic function strictly to the right of its abscissa of converge, we find that almost surely $\sum_{p} \frac{f(p)}{p^{s}}$ is a holomorphic function on the half plane $\Re(s)>1 / 2$, and so almost surely we have, for all $\Re s>\frac{1}{2}$,

$$
s \int_{1}^{\infty} \frac{\sum_{n \leq z} f(n)}{z^{s+1}} d z=\exp \left(\sum_{p} \frac{f(p)}{p^{s}}-\frac{1}{2} \sum_{p} \frac{f(p)^{2}}{p^{2 s}}+\sum_{k \geq 3} \frac{(-1)^{k+1}}{k} \sum_{p} \frac{f(p)^{k}}{p^{k s}}\right) .
$$

Next, if we write $s=\sigma+i t$ and take absolute values on both sides then we find that, almost surely,

$$
\begin{gathered}
|s| \int_{1}^{\infty} \frac{\left|\sum_{n \leq z} f(n)\right|}{z^{\sigma+1}} d z \geq \exp \left(\Re\left(\sum_{p} \frac{f(p)}{p^{s}}-\frac{1}{2} \sum_{p} \frac{f(p)^{2}}{p^{2 s}}+\sum_{k \geq 3} \frac{(-1)^{k+1}}{k} \sum_{p} \frac{f(p)^{k}}{p^{k s}}\right)\right) \\
=\exp \left(\sum_{p} \frac{\Re\left(f(p) p^{-i t}\right)}{p^{\sigma}}-\frac{1}{2} \sum_{p} \frac{\Re\left(f(p)^{2} p^{-2 i t}\right)}{p^{2 \sigma}}+O(1)\right), \quad \forall \sigma>1 / 2 .
\end{gathered}
$$

If we take $\sigma=1 / 2+1 / \log y$ for a parameter $y \geq 2$, and we note that then $|s| \asymp|t|$ provided $t \geq 1$ (say), we have almost surely that for all $y \geq 2$,

$$
\int_{1}^{\infty} \frac{\left|\sum_{n \leq z} f(n)\right|}{z^{3 / 2+1 / \log y}} d z \gg \sup _{t \geq 1} \exp \left(\sum_{p} \frac{\Re\left(f(p) p^{-i t}\right)}{p^{1 / 2+1 / \log y}}-\frac{1}{2} \sum_{p} \frac{\Re\left(f(p)^{2} p^{-2 i t}\right)}{p^{1+2 / \log y}}-\log t\right) .
$$

In the Rademacher case the first sum over $p$ is $\sum_{p} \frac{f(p) \cos (t \log p)}{p^{1 / 2+1 / \log y}}$, and (since $f(p)^{2}=$ 1) the second sum over $p$ is $\Re \sum_{p} \frac{1}{p^{1+2 / \log y+2 i t}}=\Re \log \zeta(1+2 / \log y+2 i t)+O(1)$, where $\zeta$ denotes the Riemann zeta function. Standard estimates (see e.g. Theorem 6.7 of Montgomery and Vaughan [20]) imply that $|\log \zeta(1+2 / \log y+2 i t)| \leq$ $\log \log (t+2)+O(1)$ for $t \geq 1$, so we have almost surely that for all $y \geq 2$, 


$$
\int_{1}^{\infty} \frac{\left|\sum_{n \leq z} f(n)\right|}{z^{3 / 2+1 / \log y}} d z \gg \sup _{t \geq 1} \exp \left(\sum_{p} \frac{f(p) \cos (t \log p)}{p^{1 / 2+1 / \log y}}-\log t-\log \log (t+2) / 2\right) .
$$

(The above argument and inequality (4) are essentially due to Halász [11], and are also related to the arguments of Wintner [24]. The only small difference is that Halász restricted to $1 \leq t \leq 2$. See Appendix A of Harper [12] for a presentation similar to the above.)

Now to prove Proposition 1, note that for any large parameters $x$ and $x_{0}<x_{1}$ we have

$$
\sup _{x_{0}<z<x_{1}} \frac{\mathbb{E}\left|\sum_{n \leq z} f(n)\right|}{\sqrt{z}} \geq \frac{1}{\log x} \int_{x_{0}}^{x_{1}} \frac{\mathbb{E}\left|\sum_{n \leq z} f(n)\right|}{z^{3 / 2+1 / \log x}} d z,
$$

since $\int_{x_{0}}^{x_{1}} \frac{d z}{z^{1+1 / \log x}} \leq \int_{1}^{\infty} \frac{d z}{z^{1+1 / \log x}}=\log x$. Then by Cauchy-Schwarz we always have $\mathbb{E}\left|\sum_{n \leq z} f(n)\right| \leq \sqrt{z}$, so

$$
\begin{aligned}
\int_{x_{0}}^{x_{1}} \frac{\mathbb{E}\left|\sum_{n \leq z} f(n)\right|}{z^{3 / 2+1 / \log x}} d z & \geq \int_{1}^{\infty} \frac{\mathbb{E}\left|\sum_{n \leq z} f(n)\right|}{z^{3 / 2+1 / \log x}} d z-\int_{1}^{x_{0}} \frac{d z}{z^{1+1 / \log x}}-\int_{x_{1}}^{\infty} \frac{d z}{z^{1+1 / \log x}} \\
& \geq \int_{1}^{\infty} \frac{\mathbb{E}\left|\sum_{n \leq z} f(n)\right|}{z^{3 / 2+1 / \log x}} d z-\log x_{0}-\frac{\log x}{x_{1}^{1 / \log x}} .
\end{aligned}
$$

In particular, if we choose $x_{0}=e^{\sqrt{\log x}}$ and $x_{1}=e^{(\log x) \log \log x}$, say, then we have

$$
\sup _{x_{0}<z<x_{1}} \frac{\mathbb{E}\left|\sum_{n \leq z} f(n)\right|}{\sqrt{z}} \geq \frac{1}{\log x} \int_{1}^{\infty} \frac{\mathbb{E}\left|\sum_{n \leq z} f(n)\right|}{z^{3 / 2+1 / \log x}} d z-\frac{2}{\sqrt{\log x}} .
$$

Finally, in the Rademacher case Lemma 1 implies that, with probability $1-o(1)$ as $x \rightarrow \infty$,

$\sup _{1 \leq t \leq 2(\log \log x)^{2}} \sum_{p} \frac{f(p) \cos (t \log p)}{p^{1 / 2+1 / \log x}} \geq \log \log x-\log \log \log x-O\left((\log \log \log x)^{3 / 4}\right)$.

This implies that with probability $1-o(1)$ one has

$$
\operatorname{supexp}_{t \geq 1}\left(\sum_{p} \frac{f(p) \cos (t \log p)}{p^{1 / 2+1 / \log x}}-\log t-\log \log (t+2) / 2\right) \geq \frac{\log x}{(\log \log x)^{3+o(1)}},
$$

and then by the Halász type lower bound inequality (4) we deduce

$$
\int_{1}^{\infty} \frac{\mathbb{E}\left|\sum_{n \leq z} f(n)\right|}{z^{3 / 2+1 / \log x}} d z \geq \frac{\log x}{(\log \log x)^{3+o(1)}} .
$$

Proposition 1 follows in the Rademacher case by combining this with (5). 
In the Steinhaus case the initial argument of Wintner [24] still works, so the first change that is needed in the preceding argument comes in the expression for the Euler product $F(s)$, which for $\Re(s)>1$ is now

$$
\begin{aligned}
F(s)=\prod_{p}\left(1+\sum_{j=1}^{\infty} \frac{f(p)^{j}}{p^{j s}}\right) & =\exp \left(-\sum_{p} \log \left(1-\frac{f(p)}{p^{s}}\right)\right) \\
& =\exp \left(\sum_{p} \frac{f(p)}{p^{s}}+\frac{1}{2} \sum_{p} \frac{f(p)^{2}}{p^{2 s}}+\sum_{k \geq 3} \frac{1}{k} \sum_{p} \frac{f(p)^{k}}{p^{k s}}\right) .
\end{aligned}
$$

Notice this is the same as we had in the Rademacher case, except now there are no alternating minus signs in the final exponential. The argument using the Three Series Theorem, etc. then continues as in the Rademacher case to yield that, almost surely,

$s \int_{1}^{\infty} \frac{\sum_{n \leq z} f(n)}{z^{s+1}} d z=\exp \left(\sum_{p} \frac{f(p)}{p^{s}}+\frac{1}{2} \sum_{p} \frac{f(p)^{2}}{p^{2 s}}+\sum_{k \geq 3} \frac{1}{k} \sum_{p} \frac{f(p)^{k}}{p^{k s}}\right) \quad \forall \Re(s)>1 / 2$.

Putting $s=1 / 2+1 / \log y+i t$ and taking absolute values on both sides, we deduce that almost surely,

$\int_{1}^{\infty} \frac{\left|\sum_{n \leq z} f(n)\right|}{z^{3 / 2+1 / \log y}} d z \gg \sup _{t \geq 1} \exp \left(\sum_{p}\left(\frac{\Re\left(f(p) p^{-i t}\right)}{p^{1 / 2+1 / \log y}}+\frac{1}{2} \frac{\Re\left(f(p)^{2} p^{-2 i t}\right)}{p^{1+2 / \log y}}\right)-\log t\right), \forall y \geq 2$.

Since we don't now have $f(p)^{2} \equiv 1$, we cannot remove the contribution of the prime squares using estimates for the zeta function. However, by the Steinhaus case of Lemma 1 we still have that, with probability $1-o(1)$ as $x \rightarrow \infty$,

$$
\begin{aligned}
\sup _{1 \leq t \leq 2(\log \log x)^{2}} \sum_{p}\left(\frac{\Re\left(f(p) p^{-i t}\right)}{p^{1 / 2+1 / \log x}}+\frac{1}{2} \frac{\Re\left(f(p)^{2} p^{-2 i t}\right)}{\left.p^{1+2 / \log x}\right) \geq}\right. & \log \log x-\log \log \log x \\
& -O\left((\log \log \log x)^{3 / 4}\right),
\end{aligned}
$$

and therefore with probability $1-o(1)$ we have

$$
\sup _{t \geq 1} \exp \left(\sum_{p}\left(\frac{\Re\left(f(p) p^{-i t}\right)}{p^{1 / 2+1 / \log y}}+\frac{1}{2} \frac{\Re\left(f(p)^{2} p^{-2 i t}\right)}{p^{1+2 / \log y}}\right)-\log t\right) \geq \frac{\log x}{(\log \log x)^{3+o(1)}} .
$$

Combining this estimate with (7) and (5) then proves Proposition 1 in the Steinhaus case. 


\subsection{Proofs of Theorem 1 and Proposition 2}

Proof (Proof of Theorem 1, assuming Proposition 2). In view of Proposition 2, it will suffice to prove that for all large $x$ we have

$$
\int_{1}^{\sqrt{x}}\left(\frac{\mathbb{E}\left|\sum_{n \leq z} f(n)\right|}{\sqrt{z}}\right) \frac{d z}{z} \geq \frac{\log x}{(\log \log x)^{3+o(1)}} .
$$

However, for any large parameter $y$ we have

$$
\begin{aligned}
& \int_{1}^{\sqrt{x}}\left(\frac{\mathbb{E}\left|\sum_{n \leq z} f(n)\right|}{\sqrt{z}}\right) \frac{d z}{z} \geq \int_{1}^{\sqrt{x}} \frac{\mathbb{E}\left|\sum_{n \leq z} f(n)\right|}{z^{3 / 2+1 / \log y}} d z \\
& \geq \frac{\log y}{(\log \log y)^{3+o(1)}}-\int_{\sqrt{x}}^{\infty} \frac{\mathbb{E}\left|\sum_{n \leq z} f(n)\right|}{z^{3 / 2+1 / \log y}} d z,
\end{aligned}
$$

in view of the lower bound $\int_{1}^{\infty} \frac{\mathbb{E}\left|\sum_{n \leq z} f(n)\right|}{z^{3 / 2+1 / \log y}} d z \geq \frac{\log y}{(\log \log y)^{3+o(1)}}$ obtained in (6). By Cauchy-Schwarz we always have $\mathbb{E}\left|\sum_{n \leq z} f(n)\right| \leq \sqrt{z}$, so the subtracted term here is at most

$$
\int_{\sqrt{x}}^{\infty} \frac{d z}{z^{1+1 / \log y}}=\frac{\log y}{(\sqrt{x})^{1 / \log y}} .
$$

If we choose $\log y$ somewhat smaller than $\log x$, say $\log y=(\log x) /(100 \log \log \log x)$, we deduce that

$$
\int_{1}^{\sqrt{x}}\left(\frac{\mathbb{E}\left|\sum_{n \leq z} f(n)\right|}{\sqrt{z}}\right) \frac{d z}{z} \geq \frac{\log x}{(\log \log x)^{3+o(1)}}-\frac{\log x}{(\log \log x)^{50}}=\frac{\log x}{(\log \log x)^{3+o(1)}},
$$

as required.

Proof (Proof of Proposition 2). The first part of the proof again differs slightly depending on whether we are in the Rademacher or the Steinhaus case. We will first work in the Rademacher case and then explain the small changes needed in the other situation.

Let $A_{t}:=\sum_{n \leq t} f(n)$. If we let $P(n)$ denote the largest prime factor of $n$, we have

$$
\sum_{n \leq x} f(n)=\sum_{p \leq x} \sum_{n \leq x, P(n)=p} f(n)=\sum_{p \leq x} f(p) \sum_{m \leq x / p, P(m)<p} f(m),
$$

since $f$ is multiplicative. Here the inequality $P(m)<p$ in the final sum is strict because $f$ is supported on squarefree numbers. Notice here that if $p>\sqrt{x}$ then $x / p<\sqrt{x}<p$, so we automatically have $P(m)<p$ in the inner sums over $m$. Thus we can rewrite things slightly as 


$$
\begin{aligned}
\sum_{n \leq x} f(n) & =\sum_{\sqrt{x}<p \leq x} f(p) \sum_{m \leq x / p} f(m)+\sum_{p \leq \sqrt{x}} f(p) \sum_{m \leq x / p, P(m)<p} f(m) \\
& =: \sum_{\sqrt{x}<p \leq x} f(p) A_{x / p}+B_{x},
\end{aligned}
$$

say. Notice also that the random variables $A_{x / p}$ and $B_{x}$ are independent of the $f(p)$ for $\sqrt{x}<p \leq x$.

We shall introduce a penultimate piece of notation, by defining the random variable

$$
C_{x}:=\sum_{\sqrt{x}<p \leq x} f(p) A_{x / p} .
$$

Finally, let $\varepsilon$ be a Rademacher random variable that is independent of everything else.

Now since the $(f(p))_{\sqrt{x}<p \leq x}$ are symmetric random variables independent of $B_{x}$ and the $A_{x / p}$, it follows that

$$
\sum_{n \leq x} f(n)=\sum_{\sqrt{x}<p \leq x} f(p) A_{x / p}+B_{x} \stackrel{d}{=} \varepsilon \sum_{\sqrt{x}<p \leq x} f(p) A_{x / p}+B_{x},
$$

where $\stackrel{d}{=}$ denotes equality in distribution. Then if we condition on the values of $B_{x}, C_{x}$, we find the conditional expectation

$\mathbb{E}\left(\left|\varepsilon \sum_{\sqrt{x}<p \leq x} f(p) A_{x / p}+B_{x}\right| \mid B_{x}, C_{x}\right)=(1 / 2)\left|C_{x}+B_{x}\right|+(1 / 2)\left|-C_{x}+B_{x}\right| \geq\left|C_{x}\right|$,

by the triangle inequality. Now if we average over values of $B_{x}, C_{x}$, and use the Tower Property of conditional expectations (the fact that the expectation of a conditional expectation is the unconditional expectation), we obtain

$$
\mathbb{E}\left|\sum_{n \leq x} f(n)\right|=\mathbb{E}\left|\varepsilon \sum_{\sqrt{x}<p \leq x} f(p) A_{x / p}+B_{x}\right| \geq \mathbb{E}\left|C_{x}\right| .
$$

On recalling the definitions of $C_{x}$ and $A_{x / p}$, we see we have proved the following:

Lemma 2 For all large $x$ we have

$$
\mathbb{E}\left|\sum_{n \leq x} f(n)\right| \geq \mathbb{E}\left|\sum_{\sqrt{x}<p \leq x} f(p) \sum_{m \leq x / p} f(m)\right| .
$$

(In the Steinhaus case one has a weak inequality $P(m) \leq p$ in the definition of $B_{x}$, since $f$ is totally multiplicative, but this makes no difference to the argument just given. Instead of choosing $\varepsilon$ to be a Rademacher random variable one can choose $\varepsilon$ 
to be uniformly distributed on the unit circle, and then one obtains exactly the same conclusion in Lemma 2.)

Since the $f(p)$ are Rademacher or Steinhaus random variables independent of the "coefficients" $\sum_{m \leq x / p} f(m)=A_{x / p}$, an application of Khintchine's inequality (see e.g. Gut's textbook [10]) yields that

$$
\mathbb{E}\left|\sum_{\sqrt{x}<p \leq x} f(p) \sum_{m \leq x / p} f(m)\right| \gg \mathbb{E} \sqrt{\sum_{\sqrt{x}<p \leq x}\left|\sum_{m \leq x / p} f(m)\right|^{2}} .
$$

It would be nice if we could find a way to exploit this (sharp) bound with the squares still in place on the inside, but to prove Proposition 2 we shall trade them away in order to remove the intractable squareroot. Thus by the Cauchy-Schwarz inequality and the fact that $\sum_{\sqrt{x}<p \leq x} 1 / p=\log 2+o(1)$ we have

$$
\begin{aligned}
\sum_{\sqrt{x}<p \leq x} \sqrt{\frac{1}{p}}\left|\sum_{m \leq x / p} f(m)\right| & \leq \sqrt{\sum_{\sqrt{x}<p \leq x} \frac{1}{p}} \sqrt{\sum_{\sqrt{x}<p \leq x}\left|\sum_{m \leq x / p} f(m)\right|^{2}} \\
& \ll \sqrt{\sum_{\sqrt{x}<p \leq x}\left|\sum_{m \leq x / p} f(m)\right|^{2}} .
\end{aligned}
$$

Combining this with the above, we deduce:

Lemma 3 For all large $x$ we have

$$
\mathbb{E}\left|\sum_{n \leq x} f(n)\right| \gg \sum_{\sqrt{x}<p \leq x} \frac{1}{\sqrt{p}} \mathbb{E}\left|\sum_{m \leq x / p} f(m)\right| \geq \frac{1}{\log x} \sum_{\sqrt{x}<p \leq x} \frac{\log p}{\sqrt{p}} \cdot \mathbb{E}\left|\sum_{m \leq x / p} f(m)\right| .
$$

We have now almost finished the proof of Proposition 2. If we have two primes $z \leq p \leq p^{\prime} \leq z+z / \log ^{1000} x$ for some $\sqrt{x}<z \leq x$ then

$$
\begin{aligned}
|\mathbb{E}| \sum_{m \leq x / p} f(m)|-\mathbb{E}| \sum_{m \leq x / p^{\prime}} f(m)|| & \leq \mathbb{E}\left|\sum_{x / p^{\prime}<m \leq x / p} f(m)\right| \\
& \ll \sqrt{x\left(\frac{1}{p}-\frac{1}{p^{\prime}}\right)+1} \ll \sqrt{\frac{x}{p \log ^{1000} x}}+1,
\end{aligned}
$$

by the Cauchy-Schwarz inequality and orthogonality of the $f(m)$. And we see

$$
\frac{1}{\log x} \sum_{\sqrt{x}<p \leq x} \frac{\log p}{\sqrt{p}}\left(\sqrt{\frac{x}{p \log ^{1000} x}}+1\right) \ll \frac{\sqrt{x}}{\log ^{500} x}+\frac{1}{\log x} \sum_{\sqrt{x}<p \leq x} \frac{\log p}{\sqrt{p}} \ll \frac{\sqrt{x}}{\log x},
$$


which will make a negligible contribution in Proposition 2, so in Lemma 3 we may replace each term $\mathbb{E}\left|\sum_{m \leq x / p} f(m)\right|$ by an averaged version

$$
\frac{\log ^{1000} x}{p} \int_{p}^{p\left(1+1 / \log ^{1000} x\right)} \mathbb{E}\left|\sum_{m \leq x / t} f(m)\right| d t .
$$

Since we know that primes are well distributed in intervals of relative length $1+$ $1 / \log ^{1000} x$ (with density 1 when weighted by $\log p$ ) we can rewrite Lemma 3 as

$$
\begin{aligned}
\mathbb{E}\left|\sum_{n \leq x} f(n)\right| & \gg \frac{1}{\log x} \sum_{\sqrt{x}<p \leq x} \log p \frac{\log ^{1000} x}{p} \int_{p}^{p\left(1+1 / \log ^{1000} x\right)} \mathbb{E}\left|\sum_{m \leq x / t} f(m)\right| \frac{d t}{\sqrt{t}} \\
& \gg \frac{1}{\log x} \int_{\sqrt{x}}^{x} \mathbb{E}\left|\sum_{m \leq x / t} f(m)\right| \frac{d t}{\sqrt{t}} .
\end{aligned}
$$

Proposition 2 now follows by making the substitution $z=x / t$ in the integral.

\section{Lower bounds for small moments - Proof of Theorem 2}

The proof is a very simple argument using the Cauchy-Schwarz inequality and Hölder's inequality.

Indeed, for any $0 \leq q \leq 1$ we have

$$
\begin{aligned}
\mathbb{E}\left|\sum_{n \leq N} f(n)\right| & \leq \mathbb{E}\left[\left|\sum_{n \leq N} f(n)\right|^{2 q}\right]^{1 / 2} \cdot \mathbb{E}\left[\left|\sum_{n \leq N} f(n)\right|^{2-2 q}\right]^{1 / 2} \\
& \leq \mathbb{E}\left[\left|\sum_{n \leq N} f(n)\right|^{2 q}\right]^{1 / 2} \cdot \mathbb{E}\left[\left|\sum_{n \leq N} f(n)\right|^{2}\right]^{(1-q) / 2} .
\end{aligned}
$$

Since $\mathbb{E}\left|\sum_{n \leq N} f(n)\right|^{2} \leq N$ and $\mathbb{E}\left|\sum_{n \leq N} f(n)\right| \geq \sqrt{N} /(\log \log N)^{3+o(1)}$, by re-arranging we obtain the lower bound

$$
\mathbb{E}\left[\left|\sum_{n \leq N} f(n)\right|^{2 q}\right] \geq N^{q}(\log \log N)^{-6+o(1)} .
$$

\section{Asymptotics for even moments - Proof of Theorem 3}

Note that 


$$
\begin{aligned}
\mathbb{E}\left|\sum_{n \leq X} f(n)\right|^{2 k}= & \sum_{\substack{n_{1}, \ldots, n_{k} \leq X \\
m_{1}, \ldots, m_{k} \leq X}} \mathbb{E}\left[f\left(n_{1}\right) \ldots f\left(n_{k}\right) \overline{f\left(m_{1}\right) \ldots f\left(m_{k}\right)}\right] \\
& =\sum_{\substack{n_{1}, \ldots, n_{k} \leq X \\
m_{1}, \ldots, m_{k} \leq X \\
n_{1} \ldots n_{k}=m_{1} \ldots m_{k}}} 1 .
\end{aligned}
$$

Now

$$
g\left(n_{1}, \ldots, n_{k}, m_{1}, \ldots, m_{k}\right)=\mathbf{1}_{n_{1} \ldots n_{k}=m_{1} \ldots m_{k}}
$$

is a multiplicative function of several variables 2 and our problem reduces to understanding the mean value of

$$
\sum_{\substack{n_{1}, \ldots, n_{k} \leq X \\ m_{1}, \ldots, m_{k} \leq X}} g\left(n_{1}, \ldots, n_{k}, m_{1}, \ldots, m_{k}\right)
$$

We notice that the associated multiple Dirichlet series

$$
\sum_{\substack{n_{1}, \ldots, n_{k} \\ m_{1}, \ldots, m_{k}}} \frac{g\left(n_{1}, \ldots, n_{k}, m_{1}, \ldots, m_{k}\right)}{n_{1}^{s_{1}} \ldots n_{k}^{s_{k}} m_{1}^{w_{1}} \ldots m_{k}^{w_{k}}}=\sum_{n} \sum_{n_{1} n_{2} \ldots n_{k}=n} \frac{1}{n_{1}^{s_{1}} \ldots n_{k}^{s_{k}}} \sum_{m_{1} m_{2} \ldots m_{k}=n} \frac{1}{m_{1}^{w_{1}} \ldots m_{k}^{w_{k}}}
$$

is absolutely convergent for $\mathfrak{R} s_{i}, \mathfrak{R} w_{i}>\frac{1}{2}$ and moreover it factors as

$$
H\left(s_{1}, \ldots, s_{k}, w_{1}, \ldots, w_{k}\right) \prod_{i=1}^{k} \prod_{j=1}^{k} \zeta\left(s_{i}+w_{j}\right)
$$

with $H\left(s_{1}, \ldots, s_{k}, w_{1}, \ldots, w_{k}\right)$ absolutely convergent in the region $\Re s_{i}, \Re w_{i}>\frac{1}{4}$. In addition a direct check shows that

$$
H\left(\frac{1}{2}, \ldots, \frac{1}{2}\right)=\prod_{p}\left(1-\frac{1}{p}\right)^{k^{2}} \cdot\left(1+\frac{k^{2}}{p}+\sum_{\alpha \geq 2} \frac{\left(\begin{array}{c}
a+k-1 \\
k-1
\end{array}\right)^{2}}{p^{\alpha}}\right)>0 .
$$

Therefore the main result of La Bretèche [4] is applicable with the $k^{2}$ linear forms $\ell^{(i, j)}\left(s_{1}, \ldots, s_{k}, w_{1}, \ldots, w_{k}\right):=s_{i}+w_{j}$ with $1 \leq i, j \leq k$. We note that the rank of the collection of linear forms $\ell^{(i, j)}$ (inside the space of all $\mathbb{C}$-linear forms on $\mathbb{C}^{2 k}$ ) is $2 k-1$. Therefore it follows from La Bretèche's result that $[8$ is equal to

$$
(1+o(1)) C_{k} X^{k}(\log X)^{k^{2}-(2 k-1)}
$$

\footnotetext{
${ }^{2}$ In other words

$$
g\left(n_{1}, \ldots, n_{k}, m_{1}, \ldots, m_{k}\right) g\left(u_{1}, \ldots, u_{k}, v_{1}, \ldots, v_{k}\right)=g\left(n_{1} u_{1}, \ldots, n_{k} u_{k}, m_{1} v_{1}, \ldots, m_{k} v_{k}\right)
$$
}

for any natural numbers $n_{i}, m_{i}$ and $u_{i}, v_{i}$ whose least common multiples are coprime. 
Using Théorème 2 in La Bretéche's work allows us to recover the precise value of $C_{k}$. Indeed, according to Théorème 2 in [4] we get that (8) is equal to

$$
(1+o(1)) H\left(\frac{1}{2}, \ldots, \frac{1}{2}\right) \operatorname{Vol}\left(A_{k}(X)\right)
$$

where $A_{k}(X)$ is a subset of $[1, \infty)^{k^{2}}$ corresponding to tuples $\left(a_{i, j}\right) \in[1, \infty)^{k^{2}}$ with $1 \leq i, j \leq k$ such that

$$
\begin{array}{r}
\text { for each } j \leq k: \prod_{1 \leq i \leq k} a_{i, j} \leq X \\
\text { and for each } i \leq k: \prod_{1 \leq j \leq k} a_{i, j} \leq X
\end{array}
$$

Therefore it remains to understand the asymptotic behavior of

$$
\operatorname{Vol}\left(A_{k}(X)\right)
$$

as $X \rightarrow \infty$. Surprisingly, this is somewhat involved, and the rest of the proof is devoted to that.

Proposition 3 Let $k \geq 2$ be fixed. Then,

$$
\operatorname{Vol}\left(A_{k}(X)\right) \sim\left(\begin{array}{c}
2 k-2 \\
k-1
\end{array}\right) k^{-(k-1)} \cdot \operatorname{Vol}\left(\mathscr{B}_{k}\right) \cdot X^{k} \cdot(\log X)^{(k-1)^{2}}
$$

where $\operatorname{Vol}\left(\mathscr{B}_{k}\right)$ corresponds to the $(k-1)^{2}$ dimensional volume of the Birkhoff polytope $\mathscr{B}_{k} \subset \mathbb{R}^{k^{2}}$.

The proof of the Proposition depends on the following Lemma.

Lemma 4 Let $n \geq 1$ be fixed. Then as $X \rightarrow \infty$ we have

$$
\iint_{\substack{0 \leq x_{1}, \ldots, x_{n} \leq \log X \\
0 \leq y_{1}, \ldots, y_{n} \leq \log X}} \exp \left(\min \left(x_{1}+\ldots+x_{n}, y_{1}+\ldots+y_{n}\right)\right) d x_{1} \ldots d y_{n} \sim\left(\begin{array}{c}
2 n \\
n
\end{array}\right) X^{n} .
$$

Proof. Making the substitutions $v_{i}=\log X-x_{i}$ and $w_{i}=\log X-y_{i}$ in Lemma4, we see the integral there is the same as

$$
X^{n} \iint_{\substack{0 \leq v_{1}, \ldots, v_{n} \leq \log X \\ 0 \leq w_{1}, \ldots, w_{n} \leq \log X}} \exp \left(-\max \left(v_{1}+\ldots+v_{n}, w_{1}+\ldots+w_{n}\right)\right) d v_{1} \ldots d w_{n} .
$$

Here we can extend all the ranges of integration up to positive infinity, at the cost of a multiplicative error term $1+o(1)$. Then by symmetry

$$
\begin{aligned}
& \iint_{\substack{0 \leq v_{1}, \ldots, v_{n} \\
0 \leq w_{1}, \ldots, w_{n}}} \exp \left(-\max \left(v_{1}+\ldots+v_{n}, w_{1}+\ldots+w_{n}\right)\right) d v_{1} \ldots d w_{n} \\
= & 2 \int_{0 \leq v_{1}, \ldots, v_{n}} \exp \left(-\left(v_{1}+\ldots+v_{n}\right)\right) \int_{w_{1}+\ldots+w_{n} \leq v_{1}+\ldots+v_{n}} d v_{1} \ldots d w_{n},
\end{aligned}
$$


and making the further substitution $v=v_{1}+\ldots+v_{n}$ in the integral, we see the above is

$$
\begin{aligned}
& =2 \int_{0}^{\infty} e^{-v}\left(\int_{v_{1}+\ldots+v_{n-1} \leq v} d v_{1} \ldots d v_{n-1}\right)\left(\int_{w_{1}+\ldots+w_{n} \leq v} d w_{1} \ldots d w_{n}\right) d v \\
& =2 \int_{0}^{\infty} e^{-v} v^{2 n-1}\left(\int_{v_{1}+\ldots+v_{n-1} \leq 1} d v_{1} \ldots d v_{n-1}\right)\left(\int_{w_{1}+\ldots+w_{n} \leq 1} d w_{1} \ldots d w_{n}\right) d v .
\end{aligned}
$$

Here the two integrals in brackets are simply the volume of the standard $n-1$ simplex and the standard $n$ simplex, which are well known to be $1 /(n-1)$ ! and $1 / n$ ! respectively. Threfore the above integral is equal to

$$
\frac{2}{(n-1) ! n !} \int_{0}^{\infty} e^{-v} v^{2 n-1} d v=2\left(\begin{array}{c}
2 n-1 \\
n
\end{array}\right)=\left(\begin{array}{c}
2 n \\
n
\end{array}\right) .
$$

We conclude that the integral in the statement of Lemma4 4 is equal to (as $X \rightarrow \infty$ ),

$$
(1+o(1))\left(\begin{array}{c}
2 n \\
n
\end{array}\right) X^{n}
$$

as claimed. 3.

We are now ready to prove the Proposition, and thus finish the proof of Theorem Proof (of Proposition 3). Notice first that, if we set $u_{i, j}=\log a_{i, j}$, and if we write

$$
c_{j}=\sum_{1 \leq i \leq k} u_{i, j} \text { and } r_{i}=\sum_{1 \leq j \leq k} u_{i, j}
$$

for all $i, j \leq k$, then we find

$$
\operatorname{Vol}\left(A_{k}(X)\right)=\int_{\left(u_{i, j}\right)_{1 \leq i, j \leq k} \subseteq[0, \infty)^{k^{2}}: c_{j}, r_{i} \leq \log X \forall i, j \leq k} \exp \left(\sum_{i, j \leq k} u_{i, j}\right) d u_{1,1} \ldots d u_{k, k} .
$$

To prove the proposition we shall obtain upper and lower bounds for the integral on the right that are asymptotically equal.

For convenience of writing, we start by introducing a little more notation. Let $S_{k-1}:=\sum_{i, j \leq k-1} u_{i, j}$. Let also $\mathscr{U}_{k, \varepsilon}(X)$ be the set of $u_{i, j}$ with $i, j \leq k-1$ for which

$$
\sum_{i \leq k-1} u_{i, j} \leq \log X \text { and } \sum_{j \leq k-1} u_{i, j} \leq \log X \text { and } \sum_{i, j \leq k-1} u_{i, j}>(k-2-\varepsilon) \log X .
$$

Considering the vector $\mathbf{u}$ of $u_{i, j}$ with $i, j \leq k-1$ as fixed, let $\mathscr{T}_{C, k}(\mathbf{u}, X)$ be the set of those $u_{k, i}$ with $i \leq k-1$ for which

$$
c_{j} \leq \log X \text { for all } j \leq k-1
$$


Finally, again consider the $u_{i, j}$ with $i, j \leq k-1$ as fixed let $\mathscr{T}_{R, k}(\mathbf{u}, X)$ be the set of those $u_{j, k}$ with $j \leq k-1$ for which

$$
r_{i} \leq \log X \text { for all } i \leq k-1 .
$$

We set $\varepsilon=1 / \sqrt{\log X}$, say. First seeking an upper bound, we note that if we have $S_{k-1} \leq(k-2-\varepsilon) \log X$ then $S_{k} \leq(k-\varepsilon) \log X$, and therefore the part of the integral where $S_{k-1} \leq(k-2-\varepsilon) \log X$ contributes at most

$$
X^{k-\varepsilon} \cdot \int_{\left(u_{i, j}\right)_{1 \leq i, j \leq k} \subseteq[0, \infty)^{k^{2}}: c_{j}, r_{i} \leq \log X \forall i, j \leq k} 1 d u_{1,1} \ldots d u_{k, k} \leq X^{k-\varepsilon} \log ^{k^{2}} X .
$$

This is asymptotically negligible (for any fixed $k$ ) by our choice of $\varepsilon$. Meanwhile, the part of the integral where $S_{k-1}>(k-2-\varepsilon) \log X$ is equal to

$$
\begin{aligned}
& \int_{\mathscr{U}_{k, \varepsilon}(X)} \exp \left(S_{k-1}\right) \int_{\mathscr{T}_{C, k}(\mathbf{u}, X)} \exp \left(u_{k, 1}+\ldots+u_{k, k-1}\right) \times \\
\times & \int_{\mathscr{T}_{R, k}(\mathbf{u}, X)} \exp \left(u_{1, k}+\ldots+u_{k-1, k}\right) \int_{u_{k, k}: c_{k}, r_{k} \leq \log X} \exp \left(u_{k, k}\right) d u_{1,1} \ldots d u_{k, k} .
\end{aligned}
$$

Here the innermost integral is over those

$$
0 \leq u_{k, k} \leq \log X-\max \left(u_{k, 1}+\ldots+u_{k, k-1}, u_{1, k}+\ldots+u_{k-1, k}\right),
$$

assuming the upper range of integration is at least zero. Therefore the innermost integral is certainly bounded above (extending the lower limit to negative infinity, and then performing the integration) by

$$
X \exp \left(-\max \left(u_{k, 1}+\ldots+u_{k, k-1}, u_{1, k}+\ldots+u_{k-1, k}\right)\right) .
$$

Substituting this in, it follows that (9) is less than

$$
X \int_{\mathscr{U}_{k, \varepsilon}(X)} \int_{\mathscr{T}_{C, k}(\mathbf{u}, X)} \int_{\mathscr{T}_{R, k}(\mathbf{u}, X)} \exp \left(\min \left(\sum_{1 \leq j \leq k-1} c_{j}, \sum_{1 \leq i \leq k-1} r_{i}\right)\right) \prod_{(i, j) \neq(k, k)} d u_{i, j} .
$$

At this point we change variables, letting $r_{1}, \ldots, r_{k-1}$ and $c_{1}, \ldots, c_{k-1}$ run through the interval $[0, \log X]$ so that $u_{i, k}=r_{i}-\sum_{1 \leq j \leq k-1} u_{i, j}$ and $u_{k, j}=c_{j}-\sum_{1 \leq i \leq k-1} u_{i, j}$. Since $u_{i, k} \geq 0$ and $u_{k, j} \geq 0$ this change of variable implies the additional condition that for all $i, j \leq k-1$,

$$
\sum_{j \leq k-1} u_{i, j} \leq r_{i} \text { and } \sum_{i \leq k-1} u_{i, j} \leq c_{j}
$$

The Jacobian of this linear change of variable is equal to 1 since the linear transformation taking the $\left(u_{i, j}\right)$ with $(i, j) \neq(k, k)$ into $\left(r_{\ell}, c_{\ell}, u_{i, j}\right)$ with $i, j, \ell \leq k-1$ is upper triangular with only 1's on the diagonal. 
Given $\mathbf{r}=\left(r_{1}, \ldots, r_{k-1}\right)$ and $\mathbf{c}=\left(c_{1}, \ldots, c_{k-1}\right)$ we let $\widetilde{\mathscr{U}_{k, \varepsilon}}(\mathbf{r}, \mathbf{c}, X)$ be the set of $u_{i, j}$ with $i, j \leq k-1$ satisfying the conditions (11) and the standing condition that

$$
\sum_{i, j \leq k-1} u_{i, j} \geq(k-2-\varepsilon) \log X
$$

and we let $\widetilde{\mathscr{T}_{k}}(X)$ be the set of $0 \leq r_{1}, \ldots, r_{k-1} \leq \log X$ and $0 \leq c_{1}, \ldots, c_{k-1} \leq \log X$. Then 10 can be re-written as

$$
X \int_{\widetilde{\mathscr{T}_{k}}(X)} \exp \left(\min \left(\sum_{1 \leq j \leq k-1} c_{j}, \sum_{1 \leq i \leq k-1} r_{i}\right)\right) \operatorname{Vol}\left(\widetilde{\mathscr{U}_{k, \varepsilon}}(\mathbf{r}, \mathbf{c}, X)\right) \prod_{i \leq k-1} d c_{i} d r_{i}
$$

Since $r_{i}, c_{i} \leq \log X$ for all $i \leq k-1$, we have

$$
\begin{aligned}
& \operatorname{Vol}\left(\widetilde{\mathscr{U}_{k, \varepsilon}}(\mathbf{r}, \mathbf{c}, X)\right) \leq \operatorname{Vol}\left(\widetilde{\mathscr{U}_{k, \varepsilon}}(\log \mathbf{X}, \log \mathbf{X}, \mathbf{X})\right) \\
& \quad=(\log X)^{(k-1)^{2}} \cdot \operatorname{Vol}\left(\widetilde{\mathscr{U}_{k, \varepsilon}}(\mathbf{1}, \mathbf{1}, e)\right) \sim(\log X)^{(k-1)^{2}} \cdot \operatorname{Vol}\left(\widetilde{\mathscr{U}_{k, 0}}(\mathbf{1}, \mathbf{1}, e)\right)
\end{aligned}
$$

as $X \rightarrow \infty$, where $\log \mathbf{X}:=(\log X, \ldots, \log X)$ and $\mathbf{1}:=(1, \ldots, 1)$, and where we recall for the final asymptotic that $\varepsilon=1 / \sqrt{\log X}$. As already mentioned in the introduction $\operatorname{Vol}\left(\widetilde{\mathscr{U}_{k, 0}}(\mathbf{1}, \mathbf{1}, e)\right)=k^{-(k-1)} \operatorname{Vol}\left(\mathscr{B}_{k}\right)$ where $\mathscr{B}_{k}$ is the Birkhoff polytope. It follows that 10] is

$$
\begin{aligned}
\leq(1+o(1)) X(\log X)^{(k-1)^{2}} & \cdot k^{-(k-1)} \operatorname{Vol}\left(\mathscr{B}_{k}\right) \\
& \times \int_{\widetilde{\mathscr{T}_{k}}(X)} \exp \left(\min \left(\sum_{1 \leq j \leq k-1} c_{j}, \sum_{1 \leq i \leq k-1} r_{i}\right)\right) \prod_{i \leq k-1} d c_{i} d r_{i}
\end{aligned}
$$

and by Lemma 4 this is less than or equal to to

$$
(1+o(1)) X(\log X)^{(k-1)^{2}} \cdot k^{-(k-1)} \operatorname{Vol}\left(\mathscr{B}_{k}\right) \cdot X^{k-1}\left(\begin{array}{c}
2 k-2 \\
k-1
\end{array}\right)
$$

thus finishing the proof of the upper bound.

For the lower bound we restrict attention, as we may (due to positivity), to the part of the integral where $S_{k-1}>(k-2+\varepsilon) \log X$ and each $r_{i}, c_{i}$ is $\geq(1-\varepsilon) \log X$. The point of the former condition is that if it is satisfied then

$$
u_{1, k}+u_{2, k}+\ldots+u_{k-1, k} \leq(k-1) \log X-S_{k-1} \leq(1-\varepsilon) \log X
$$

and similarly $u_{k, 1}+u_{k, 2}+\ldots+u_{k, k-1} \leq(1-\varepsilon) \log X$, and therefore

$$
\log X-\max \left(u_{k, 1}+\ldots+u_{k, k-1}, u_{1, k}+\ldots+u_{k-1, k}\right)>\varepsilon \log X=\sqrt{\log X} \rightarrow \infty .
$$

Therefore arguing as above the innermost integral over $u_{k, k}$ in (9) contributes

$$
(1+o(1)) X \exp \left(-\max \left(u_{k, 1}+\ldots+u_{k, k-1}, u_{1, k}+\ldots+u_{k-1, k}\right)\right) .
$$


Proceeding as before we thus arrive to (13) but with the additional condition that $(1-\varepsilon) \log X<r_{i}, c_{i}<\log X$ (and with the condition that $\sum_{i, j \leq k-1} u_{i, j} \geq(k-2-$ $\varepsilon) \log X$ replaced by the condition that $\left.\sum_{i, j \leq k-1} u_{i, j} \geq(k-2+\varepsilon) \log X\right)$. It follows that on this set of $r_{i}$ and $c_{i}$ we have

$$
\begin{aligned}
\operatorname{Vol}\left(\widetilde{\mathscr{U}}_{k, \varepsilon}(\mathbf{r}, \mathbf{c}, X)\right)>\operatorname{Vol}\left(\widetilde{\mathscr{U}}_{k, \varepsilon}((\mathbf{1}-\varepsilon) \log \mathbf{X},(\mathbf{1}-\varepsilon) \log \mathbf{X}, X)\right) \\
=(1+o(1))(\log X)^{(k-1)^{2}} \cdot k^{-(k-1)} \operatorname{Vol}\left(\mathscr{B}_{k}\right)
\end{aligned}
$$

Therefore we obtained the following lower bound

$$
\begin{aligned}
&(1+o(1)) X(\log X)^{(k-1)^{2}} \cdot k^{-(k-1)} \operatorname{Vol}\left(\mathscr{B}_{k}\right) \\
& \times \iint_{\widetilde{\mathscr{T}}_{k, \varepsilon}(X)} \exp \left(\min \left(\sum_{1 \leq j \leq k-1} c_{j}, \sum_{1 \leq i \leq k-1} r_{i}\right)\right) \prod_{i \leq k-1} d c_{i} d r_{i}
\end{aligned}
$$

where $\widetilde{\mathscr{T}}_{k, \varepsilon}(X)$ is the set of $r_{i}, c_{i}$ satisfying $(1-\varepsilon) \log X<r_{i}, c_{i} \leq \log X$ for all $i \leq k-1$. Note that the condition $(1-\varepsilon) \log X<r_{i}, c_{i}$ can be dropped. Indeed the contribution to the integral of any tuple of $\left(r_{1}, \ldots, r_{k-1}\right)$ or $\left(c_{1}, \ldots, c_{k-1}\right)$ where at least one of the $c_{i}, r_{i}$ is $\leq(1-\varepsilon) \log X$ is $\leq X^{k-1-\varepsilon}$ and therefore negligible. Thus we can extend the integration to all of $c_{i}, r_{i} \leq \log X$. Because of this Lemma 4 is applicable and we have therefore obtained the lower bound

$$
\geq(1+o(1))\left(\begin{array}{c}
2 k-2 \\
k-1
\end{array}\right) \cdot k^{-(k-1)} \operatorname{Vol}\left(\mathscr{B}_{k}\right) X^{k} \cdot(\log X)^{(k-1)^{2}}
$$

as claimed. Since we have obtained asymptotically matching upper and lower bounds the proof of the proposition is finished.

\section{Proof of Theorem 4}

In the Rademacher case we have, letting $\square$ denote a generic square,

$$
\begin{aligned}
\mathbb{E}\left(\sum_{n \leq X} f(n)\right)^{k} & =\sum_{n_{1}, \ldots, n_{k} \leq X} \mathbb{E}\left[f\left(n_{1}\right) \ldots f\left(n_{k}\right)\right] \\
& =\sum_{\substack{n_{1}, \ldots, n_{k} \leq X \\
n_{1} \ldots n_{k}=\square}} \mu^{2}\left(n_{1}\right) \ldots \mu^{2}\left(n_{k}\right)
\end{aligned}
$$

Let $g\left(n_{1}, \ldots, n_{k}\right)$ be a multiplicative function of several variables, supported on square-free $n_{i}$, and such that $g=1$ when $n_{1} \ldots n_{k}=\square$ and $g=0$ otherwise. Then we find that the Dirichlet series

$$
\sum_{n_{1}=1}^{\infty} \ldots \sum_{n_{k}=1}^{\infty} \frac{g\left(n_{1}, \ldots, n_{k}\right)}{n_{1}^{s_{1}} \ldots n_{k}^{s_{k}}}
$$


is equal to

$$
\prod_{p}\left(1+\sum_{\substack{0 \leq \alpha_{1}, \ldots, \alpha_{k} \leq 1 \\ \alpha_{1}+\ldots+\alpha_{k} \equiv 0 \bmod 2}} \frac{1}{p^{\alpha_{1} s_{1}+\ldots+\alpha_{k} s_{k}}}\right)
$$

This factors as

$$
H\left(s_{1}, \ldots, s_{k}\right) \prod_{1 \leq i<j \leq k} \zeta\left(s_{i}+s_{j}\right)
$$

with

$$
H\left(\frac{1}{2}, \ldots, \frac{1}{2}\right)=\prod_{p}\left(1-\frac{1}{p}\right)^{k(k-1) / 2}\left(1+\sum_{1 \leq j \leq k / 2} \frac{\left(\begin{array}{c}
k \\
2 j
\end{array}\right)}{p^{j}}\right)
$$

The main result of La Bretèche is applicable with $\left(\begin{array}{c}k \\ 2\end{array}\right)$ linear forms $\ell^{(i, j)}\left(s_{1}, s_{2}, \ldots, s_{k}\right)=$ $s_{i}+s_{j}$ defined for $1 \leq i<j \leq k$. The rank of these linear forms is equal to $k$ for $k \geq 3$ (for $k=2$ the rank is equal to 1 since there is only one form in that case). Therefore applying La Bretèche's result it follows that the moment is asymptotically

$$
(1+o(1)) C_{k} X^{k / 2}(\log X)^{\left(\begin{array}{l}
k \\
2
\end{array}\right)-k} .
$$

In order to determine the constant $C_{k}$ one could use Théorème 2 of La Bretèche, to conclude that the moment is asymptotically

$$
(1+o(1)) H\left(\frac{1}{2}, \ldots, \frac{1}{2}\right) \operatorname{Vol}(B(X))
$$

where $B(X)$ is the set of $\left(u_{i, j}\right)_{i<j} \in \mathbb{R}^{k(k-1) / 2}$ such that

$$
\text { for all } 1 \leq i \leq k: \prod_{j<i} u_{j, i} \prod_{i<j} u_{i, j} \leq X
$$

and then proceed in a manner similar to Theorem 3. However we leave this computation to the interested reader.

\section{References}

1. M. Beck, D. Pixton. The Erhart Polynomial of the Birkhoff polytope. Discrete Comput. Geom., 30, no. 4, pp 623-637. 2003

2. A. Bondarenko, W. Heap, K. Seip. An inequality of Hardy-Littlewood type for Dirichlet polynomials. J. Number Theory, 150, pp 191-205. 2015

3. A. Bondarenko, K. Seip. Helson's problem for sums of a random multiplicative function. Available online at arxiv: 1411.6388

4. R. de la Bretèche. Estimation de sommes multiples de fonctions arithmétiques. Compositio Math., 128, no. 3, pp. 261-298, 2001.

5. E. R. Canfield, B. D. McKay. The asymptotic volume of the Birkhoff polytope. Online J. Anal. Comb., no. 4, 4 pp., 2009

6. C. S. Chan, D. P. Robbins. On the volume of the polytope of doubly stochastic matrices. Experiment. Math., 8, no. 3, pp. 291-300, 1999 
7. S. Chatterjee, K. Soundararajan. Random multiplicative functions in short intervals. Int. Math. Res. Not., pp 479-492. 2012

8. B. Conrey, A. Gamburd. Pseudomoments of the Riemann zeta function and pseudomagic squares. J. Number Theory, 117, no. 2, pp 263-278. 2006

9. B. Conrey, S. Gonek. High moments of the Riemann zeta-function. Duke Math. J., 107, no. 3, pp. 577-604, 2001

10. A. Gut. Probability: A Graduate Course. Second edition, published by Springer Texts in Statistics. 2013

11. G. Halász. On random multiplicative functions. In Hubert Delange Colloquium, (Orsay, 1982). Publications Mathématiques d'Orsay, 83, pp 74-96. Univ. Paris XI, Orsay. 1983

12. A. J. Harper. Bounds on the suprema of Gaussian processes, and omega results for the sum of a random multiplicative function. Ann. Appl. Probab., 23, no. 2, pp 584-616. 2013

13. A. J. Harper. On the limit distributions of some sums of a random multiplicative function. Journal für die reine und angewandte Mathematik, 678, pp 95-124. 2013

14. A. J. Harper. A note on the maximum of the Riemann zeta function, and log-correlated random variables. Available on-line at arxiv:1304:0677

15. W. Heap, S. Lindqvist. Moments of random multiplicative functions and truncated characteristic polynomials. Preprint.

16. H. Helson. Hankel Forms. Studia Math., 198, no. 1, pp. 79-84, 2010.

17. B. Hough. Summation of a random multiplicative function on numbers having few prime factors. Math. Proc. Cambridge Philos. Soc., 150, pp 193-214. 2011

18. Y-K. Lau, G. Tenenbaum, J. Wu. On mean values of random multiplicative functions. Proc. Amer. Math. Soc., 141, pp. 409-420, 2013

19. S. R. Louboutin, M. Munsch. The second and fourth moments of theta functions at their central point. J. Number Theory, 133, no. 4, pp. 1186-1193, 2013

20. H. L. Montgomery, R. C. Vaughan. Multiplicative Number Theory I: Classical Theory. First edition, published by Cambridge University Press. 2007

21. N. Ng. The distribution of the summatory function of the Möbius function. Proc. London. Math. Soc., 89, no. 3, pp. 361-389, 2004.

22. J. Ortegà-Cerda, K. Seip. A lower bound in Nehari's theorem on the polydisc. J. Anal. Math., 118, no. 1, pp. 339-342, 2012.

23. I. Pak. Four questions on Birkhoff polytope. Annals of Combinatorics, 4, pp. 83-90, 2000.

24. A. Wintner. Random factorizations and Riemann's hypothesis. Duke Math. J., 11, pp 267-275. 1944 